\title{
Comparing Epidural Analgesia and ON-Q Infiltrating Catheters for Pain Management after Hepatic Resection
}

\author{
Jose M. Soliz ${ }^{1}$, Rodolfo Gebhardt ${ }^{1}$, Lei Feng ${ }^{1}$, Wenli Dong ${ }^{1}$, Margaret Reich ${ }^{2}$, Steven Curley ${ }^{1}$ \\ ${ }^{1}$ The University of Texas MD Anderson Cancer Center, Houston, USA; ${ }^{2}$ The University of Texas Health Science Center, San Anto- \\ nio Medical School, San Antonio, USA. \\ Email: jsoliz@mdanderson.org
}

Received October $20^{\text {th }}, 2012$; revised November $25^{\text {th }}, 2012$; accepted December $12^{\text {th }}, 2012$

\begin{abstract}
Background and Objectives: Pain control after hepatic resection presents unique challenges as subcostal incisions, rib retraction, and diaphragmatic irritation can lead to significant pain. Both epidural analgesia and ON-Q catheters have been used for postoperative pain management after hepatic surgery, but to our knowledge have not been directly compared. Methods: The records of 143 patient between the ages 18 and 70 were reviewed who underwent hepatic resection by a single surgeon. Patients were categorized according to method of postoperative pain control. Average pain scores for both study groups were collected until POD\#3. Results: Demographic data and the length of surgery were similar between the groups (all $P>0.05$ ). On the day of surgery and POD\#1, average pain scores for the epidural group were lower than the ON-Q group $(P<0.0001$ and $P=0.0008$ respectively). There was no difference in pain scores on POD $\# 2(P=0.2369)$ or POD\#3 $(P=0.2289)$. Conclusions: Epidural analgesia provides superior pain control on the day of surgery and POD\#1 when compared to On-Q catheter with IV PCA. There was no difference in pain scores on POD\#2 or POD\#3. Future prospective randomized trials comparing these analgesic methods will be required to further evaluate enhanced recovery after hepatic surgery.
\end{abstract}

Keywords: Hepatic Resection; Epidural; On-Q Catheter; Postoperative Pain Control

\section{Introduction}

Optimal pain management after hepatic resection presents unique challenges as subcostal incisions, visceral pain, rib retraction, and diaphragmatic irritation can lead to significant postoperative pain.

A variety of techniques have been used to provide pain relief after hepatic resection including epidural analgesia [1-3], intravenous patient controlled analgesia [2], and local anesthesia infiltration catheters [4]. To our knowledge, local anesthetic infiltrating catheters (ON-Q catheters) with the concurrent use of intravenous patient controlled analgesia have not been directly compared to epidural analgesia for pain management after hepatic surgery. We conducted a retrospective study to evaluate these methods of postoperative pain management in patients who received subcostal incisions for hepatic tumor resection.

\section{Methods}

After approval from the Institutional Review Board (University of Texas MD Anderson Cancer Center, Houston, TX), we reviewed the records of 143 patients who underwent hepatic resection by a single surgeon at MD
Anderson Cancer Center between July 2005 and July 2010. The Institutional Review Board waived the requirement for consent. Patients included in this study were ages 18 to 70 , and were categorized according to method of postoperative pain control. The two study groups received thoracic epidural analgesia (TEA) or local anesthetic infiltrating catheter (ON-Q Pain Buster; I-Flow Corporation, Lake Forest, CA) plus intravenous patient controlled analgesia (IV PCA) for postoperative pain control. We excluded patients whose surgery was emergent. A right subcostal or bilateral subcostal incision was performed for surgical exposure for each patient. Pain scores were obtained from each patient using an 11 point visual analog scale $(0-10)$ by the bedside nurse every four hours. Average pain scores for each day were collected.

\subsection{Epidural Analgesia Group}

Thoracic epidural catheters were placed by the anesthesiologist after informed consent was obtained. All epidurals were placed in the thoracic T6 - T10 region, and were managed postoperatively by a 24-hour acute pain service. Epidural infusions were typically started intra- 
operatively and maintained until the patient was tolerating a full liquid diet. The epidural catheter insertion site, neurologic exam, and motor exam were assessed during each shift by our 24-hour acute pain service. Epidural solution included a combination of local anesthetic and narcotic (bupivicaine $0.075 \%$ or $0.1 \%$, with hydromorphone $5 \mathrm{mcg} / \mathrm{cc}$ or $2 \mathrm{mcg} / \mathrm{cc}$ ). All patients had a basal infusion of the epidural solution and a patient controlled bolus (PCEA). Patients who experienced persistent nausea, drowsiness, or pruritis despite rescue medication had the epidural narcotic decreased incrementally until the side effect subsided.

\subsection{ON-Q Catheter Group}

Patients who received an ON-Q local anesthetic infiltrating catheter had the catheter placed intraoperatively by the surgical team. Each ON-Q catheter consisted of two 10 inch multiorifice tubes that were inserted above the fascia, one tube placed on each side of the surgical incision. Each catheter was bolused with 10 cc of a 1:1 mixture of $1 \%$ lidocaine and $0.25 \%$ bupivicaine prior to skin closure. After skin closure, the ON-Q pump containing $0.5 \%$ ropivicaine was connected and delivered 2 cc of local anesthetic per hour thru each of the 2 catheters. Patients were also given an IV PCA, commonly morphine $1 \mathrm{mg}$ every 10 minutes prn without a basal infusion, which was started immediately in the post anesthetic care unit. Management of the IV PCA in the ON-Q group was performed by the primary surgical team. The ON-Q catheters were maintained in place until postoperative day 3 , at which time they were removed.

\subsection{Statistical Analysis}

The patients' demographic and clinical characteristics were collected, including age, gender, body mass index (BMI), American Society of Anesthesiology (ASA) physical status classification score, length of surgery, type of surgical incision (unilateral vs. bilateral), estimated surgical blood loss, time to first bowel movement, number of blood draws to test coagulation status (i.e., prothrombin time, partial thromboplastin time, International Normalized Ratio, and platelet count), and length of hospital stay.

Frequencies and percentages were reported for categorical variables (such as gender and ASA category). Mean and standard deviation (std) were provided for continuous data (such as age and BMI). A Wilcoxon's rank sum test was used to compare differences in continuous variables between epidural and ON-Q groups. Chi square test was used to compare categorical variables between groups. For the primary endpoint, pain scores, repeated measures models were fitted to assess the treatment effect and time effect. Means and standard deviations for daily pain scores over time were provided for the epidural analgesia and the ON-Q groups. All tests were two-sided. $\mathrm{P}$ values less than 0.05 were considered statistically significant. All analyses were conducted using SAS (version 9.1, Cary, NC) and S-plus (TIBCO Software Inc., Palto Alto, CA) statistical software.

\section{Results}

\subsection{Patient Characteristics}

Demographic data for all 143 patients are shown in Table 1. The epidural group were $50.7 \%$ male, $49.3 \%$ female, The ON-Q group were $51.4 \%$ male, $48.6 \%$ female. Age, gender, BMI, and ASA scores were not statistically different between the two study groups $(P>0.05)$. Length of surgery and estimated surgical blood loss (EBL) were also not different between the study groups (all $P>0.05$ ).

\subsection{Epidural Analgesia Group}

A total of 71 consecutive patients who received epidural analgesia were analyzed. All epidurals were placed by an attending anesthesiologist in a thoracic T6 to T10 interspace. The epidurals were managed by our 24-hour acute pain service headed by a faculty anesthesiologist. Epidural catheters were maintained for an average $3.5 \pm 1.2$ days. No epidural related complications were reported.

\subsection{ON-Q Catheter Group}

A total of 72 consecutive patients receiving a local anesthetic infiltrating catheter and an IV PCA were included for analysis. $63.8 \%$ of patients $(n=46)$ received a morphine IV PCA, while $36.1 \%(n=26)$ received a hydromorphone IV PCA. All ON-Q catheters were removed on postoperative day \#3 by the surgical team. No ON-Q catheter related complications were reported.

\subsection{Pain Analysis}

The mean daily pain scores for each study group is graphically depicted over time in Figure 1. The treatment effect evaluated by the repeated measures model was significant $(P<0.0001)$. The time effect fitted as a categorical variable was significant $(P$ value $<0.0001)$. The interaction effect between treatment and time was also significant $(P$ value $<0.0001)$. On the day of surgery and postoperative day 1 , mean pain scores for the epidural group were statistically lower $(P<0.001$ and $P=$ 0.0008 respectively) than the ON-Q group (Table 2). There was no statistical difference $(P>0.05)$ in mean pain scores between the 2 study groups on postoperative days 2 and 3 (Table 2).

\subsection{Laboratory Blood Draws}

The number of coagulation lab draws (PT, PTT, INR, 
Table 1. Patient factors and demographic data.

\begin{tabular}{cccc}
\hline Characteristic & $\begin{array}{c}\text { Epidural } \\
\text { Analgesia }\end{array}$ & ON-Q Catheter & $P$ value \\
\hline Age (mean \pm std) & $54.3 \pm 9.9$ & $53.7 \pm 11.2$ & 0.9101 \\
Sex $\quad \begin{array}{c}\text { Male } \\
\text { female }\end{array}$ & $\begin{array}{c}36(50.7 \%) \\
35(49.3 \%)\end{array}$ & $37(51.4 \%)$ & 0.9347 \\
BMI (mean \pm std) & $29.1 \pm 5.1$ & $28.2 \pm 4.7$ & 0.2509 \\
ASA $\quad 2$ & $22(31.0 \%)$ & $13(18.1 \%)$ & 0.0722 \\
score $\quad 3$ & $49(69.0 \%)$ & $59(81.9 \%)$ & \\
$\begin{array}{c}\text { Length of Surgery } \\
\text { (hours; mean } \pm \text { std ) }\end{array}$ & $2.0 \pm 0.7$ & $1.9 \pm 0.6$ & 0.2247 \\
$\begin{array}{c}\text { Estimated Surgical } \\
\text { Blood Loss } \\
\text { (cc } \pm \text { std) }\end{array}$ & $353.7 \pm 306.5$ & $376.3 \pm 413.3$ & 0.9887 \\
$\begin{array}{c}\text { Time to Bowel } \\
\text { Movement } \\
\text { (days; mean } \pm \text { std) }\end{array}$ & $4.5 \pm 2.2$ & $5.2 \pm 2.9$ & 0.1209 \\
$\begin{array}{c}\text { Length of Hospital Stay } \\
\text { (days; mean } \pm \text { std) }\end{array}$ & $8.5 \pm 5.6$ & $7.8 \pm 5.5$ & 0.4580 \\
\hline
\end{tabular}

Table 2. Mean daily pains scores.

\begin{tabular}{cccc}
\hline Day & $\begin{array}{c}\text { Epidural } \\
\text { mean } \pm \text { std }\end{array}$ & $\begin{array}{c}\text { ON-Q Catheter } \\
\text { mean } \pm \text { std }\end{array}$ & $P$ value \\
\hline Day of Surgery & $2.1 \pm 1.4$ & $4.3 \pm 1.6$ & $P<0.0001$ \\
POD\#1 & $1.8 \pm 1.2$ & $2.6 \pm 1.5$ & $P=0.0008$ \\
POD\#2 & $1.1 \pm 1.0$ & $1.4 \pm 1.3$ & $P=0.2369$ \\
POD\#3 & $1.0 \pm 1.1$ & $1.2 \pm 1.3$ & $P=0.2289$ \\
\hline
\end{tabular}

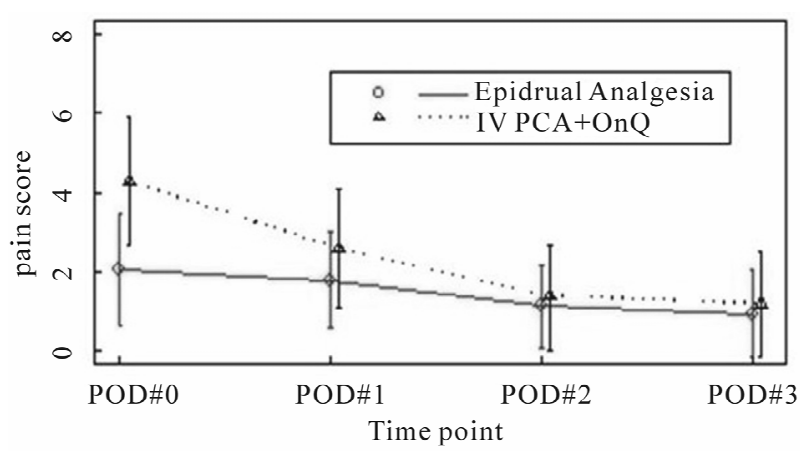

Figure 1. mean pain score by treatment group.

CBC, and platelets) for each patient until time of discharge was collected. The calculated mean of the total number of lab draws per patient in the epidural group ( $\mathrm{n}=$ $14.2 \pm 19.6)$ was not significantly different $(P=0.1020)$ than the ON-Q group $(\mathrm{n}=14.0 \pm 8.4)$.

\subsection{Length of Hospital Stay}

The length of hospital stay for the epidural group ranged from 3 to 42 days. The length of hospital stay for the ON-Q catheter group ranged from 4 to 49 days. There was no difference $(P=0.4580)$ in the mean length of hospital stay between the epidural group (8.5 \pm 5.6 days) and the ON-Q group ( $7.8 \pm 5.5$ days).

\subsection{Time to First Bowel Movement}

Overall, the time to first bowel movement was $4.9 \pm 2.6$ days. The time to first bowel movement in the epidural analgesia group ( $4.5 \pm 2.2$ days) was not statistically different than the ON-Q catheter group (5.2 \pm 2.9 days) $(P=$ 0.1209). There was also no difference in time to first bowel movement when comparing patients who received epidural narcotic hydromorphone $5 \mathrm{mcg} / \mathrm{cc}$ versus hydromorphone $2 \mathrm{mcg} / \mathrm{cc},(P=0.73)$.

\section{Discussion}

Adequate perioperative pain control is imperative in decreasing perioperative complications. In previous studies of epidural analgesia for abdominal surgery, patients with epidurals have better postoperative pain control [5-8], sooner return of bowel function [9,10], earlier ambulation, and fewer pulmonary complications [11,12] than patients whom received IV PCA. The use of multimodal pain therapy further reduces postoperative pain while reducing opioid related side effects [3,13].

The use of epidural analgesia for postoperative pain control after hepatic resection has its benefits, but concerns about postoperative coagulation abnormalities in patients undergoing major hepatectomy have many centers reluctant to use epidurals routinely. Alterations in postoperative coagulations profile after hepatic resection occur frequently [14-16]. In a study by Shontz et al., $47 \%$ of the patients who underwent hepatic resection had at least 1 abnormal coagulation lab result during the postoperative period [16]. Weinberg et al demonstrated increased prothrombin times after hepatic resection correlating with length of surgery and the weight of the resected specimen [17]. As alterations in coagulation are a possibility after liver resection, regular assessment and early recognition of complications are imperative $[18,19]$. At our institution, each patient who received epidural analgesia is evaluated regularly after surgery for neurologic deficits, side effects to pain therapy, motor status, sensory status, and the catheter entry site is assessed. Coagulation profiles are reviewed prior to epidural catheter removal, and neurological checks are performed for 48 hours after epidural removal. In our study, there were no differences in the number of coagulation lab draws between the epidural analgesia and ON-Q catheter groups.

Local anesthetic infiltrating catheters have been used for many types of surgery [4,13,20-22]. A systematic review and meta-analysis of local anesthetic wound infusion catheters for colorectal surgery showed that their use was associated with significantly reduced opioid 
consumption [21]. In a prospective study of the use ON-Q local anesthetic infiltrating catheters for open nephrectomy, postoperative narcotic consumption was reduced, and patients had earlier return of bowel function and more rapid discharge from the hospital [13]. In a preliminary study using continuous intramuscular bupivicaine after hepatic resection, postoperative pain scores were satisfactory [4]. In our study, pain scores were significantly lower in the epidural analgesia group than in the ON-Q catheter group on the day of surgery and postoperative day 1 . It is noteworthy that $71.8 \%$ of the patients in the epidural group versus only $22.2 \%$ of patients in the ON-Q catheter group had a bilateral subcostal incision. It is possible that a larger incision would lead to more postoperative pain. Thus, had each group had the same proportion of patients with a bilateral subcostal incision, pain scores might have been even more favorable for the epidural analgesia group.

\section{Conclusion}

This study demonstrates that epidural analgesia provides better pain control early in the postoperative course compared to use of an ON-Q catheter with IV PCA. However, there was no difference in the time to first bowel movement, time to discharge, or number of coagulation lab draws. ON-Q catheters with IV PCA provide a good alternative to postoperative pain control for hepatic resection when epidural catheter placement is not feasible. Future prospective randomized trials comparing epidural analgesia and use of ON-Q catheters will be required to evaluate outcomes and enhanced recovery after hepatic surgery.

\section{Acknowledgements}

The authors thank Chris Rubio, MBA (Department of Anesthesiology and Perioperative Medicine, MD Anderson Cancer Center) for assistance in data collection, and Stephanie Deming, Scientific Editor (Scientific Publications, MD Anderson Cancer Center), for editing this article.

\section{REFERENCES}

[1] P. Feltracco, M. L. Brezzi, S. Barbieri, E. Serra, M. Milevoj and C. Ori, "Epidural Anesthesia and Analgesia in Liver Resection and Living Donor Hepatectomy," Transplantation Proceedings, Vol. 40, No. 4, 2008, pp. 1165-1168. doi:10.1016/j.transproceed.2008.03.108

[2] A. Page, B. Rostad, C. A. Staley, J. H. Levy, J. Park, M. Goodman, et al., "Epidural Analgesia in Hepatic Resection," Journal of the American College of Surgeons, Vol. 206, No. 3, 2008, pp. 1184-1192. doi:10.1016/j.jamcollsurg.2007.12.041

[3] J. B. Cywinski, B. M. Parker, M. Xu and S. A. Irefin, “A
Comparison of Postoperative Pain Control in Patients after Right Lobe Donor Hepatectomy and Major Hepatic Resection for Tumor," Anesthesia \& Analgesia, Vol. 99, No. 6, 2004, pp. 1747-1752. doi:10.1213/01.ANE.0000136423.17446.5D

[4] S. Basu, A. Tamijmarane, D. Bulters, J. K. Wells, T. G. John and M. Rees, "An Alternative Method of Wound Pain Control Following Hepatic Resection: A Preliminary Study,” HPB (Oxford), Vol. 6, No. 3, 2004, pp. 186-189. doi:10.1080/13651820410030844

[5] M. Ali, D. C. Winter, A. M. Hanly, C. O’Hagan, J. Keaveny and P. Broe, "Prospective, Randomized, Controlled Trial of Thoracic Epidural or Patient-Controlled Opiate Analgesia on Perioperative Quality of Life," British Journal of Anaesthesia, Vol. 104, No. 3, 2010, pp. 292297. doi:10.1093/bja/aeq006

[6] C. L. Wu, S. R. Cohen, J. M. Richman, A. J. Rowlingson, G. E. Courpas, K. Cheung, et al., "Efficacy of Postoperative Patient-Controlled and Continuous Infusion Epidural Analgesia versus Intravenous Patient-Controlled Analgesia with Opioids: A Meta-Analysis,” Anesthesiology, Vol. 103, No. 5, 2005, pp. 109-110. doi:10.1097/00000542-200511000-00023

[7] S. J. Dolin, J. N. Cashman and J. M. Bland, "Effectiveness of Acute Postoperative Pain Management: I. Evidence from Published Data,” British Journal of Anaesthesia, Vol. 89, No. 3, 2002, pp. 409-423.

[8] B. M. Block, S. S. Liu, A. J. Rowlingson, A. R. Cowan, J. A. Cowan Jr. and C. L. Wu, "Efficacy of Postoperative Epidural Analgesia: A Meta-Analysis,” JAMA, Vol. 290, No. 18, 2003, pp. 2455-2463. doi:10.1001/jama.290.18.2455

[9] H. Jorgensen, J. Wetterslev, S. Moiniche and J. B. Dahl, "Epidural Local Anaesthetics versus Opioid-Based Analgesic Regimens on Postoperative Gastrointestinal Paralysis, PONV and Pain after Abdominal Surgery," Cochrane Database of Systematic Reviews, No. 4, 2000, Article ID: CD001893.

[10] H. Kehlet and J. B. Dahl, “Anaesthesia, Surgery, and Challenges in Postoperative Recovery,” Lancet, Vol. 362, No. 9399, 2003, pp. 1921-1928. doi:10.1016/S0140-6736(03)14966-5

[11] S. S. Liu and C. L. Wu, "Effect of Postoperative Analgesia on Major Postoperative Complications: A Systematic Update of the Evidence," Anesthesia \& Analgesia, Vol. 104, No. 3, 2007, pp. 689-702. doi:10.1213/01.ane.0000255040.71600.41

[12] J. R. Rigg, K. Jamrozik, P. S. Myles, B. S. Silbert , P. J. Peyton, R. W. Parsons, et al., "Epidural Anaesthesia and Analgesia and Outcome of Major Surgery: A Randomised Trial,” Lancet, Vol. 359, No. 9314, 2002, pp. 1276-1282. doi:10.1016/S0140-6736(02)08266-1

[13] E. Forastiere, M. Sofra, D. Giannarelli, L. Fabrizi and G. Simone, "Effectiveness of Continuous Wound Infusion of 0.5\% Ropivacaine by On-Q Pain Relief System for Postoperative Pain Management after Open Nephrectomy," British Journal of Anaesthesia, Vol. 101, No. 6, 2008, pp. 841-847. doi:10.1093/bja/aen309

[14] J. J. Pelton, J. P. Hoffman and B. L. Eisenberg, “Com- 
parison of Liver Function Tests after Hepatic Lobectomy and Hepatic Wedge Resection,” The American Journal of Surgery, Vol. 64, No. 5, 1998, pp. 408-414.

[15] D. M. Stamenkovic, Z. B. Jankovic, G. J. Toogood, J. P. Lodge and M. C. Bellamy, "Epidural Analgesia and Liver Resection: Postoperative Coagulation Disorders and Epidural Catheter Removal,” Minerva Anestesiologica, Vol. 77, No. 7, 2008, pp. 671-679.

[16] R. Shontz, V. Karuparthy, R. Temple and T. J. Brennan, "Prevalence and Risk Factors Predisposing to Coagulopathy in Patients Receiving Epidural Analgesia for Hepatic Surgery,” Regional Anesthesia and Pain Medicine, Vol. 34, No. 4, 2009, pp. 308-311. doi:10.1097/AAP.0b013e3181ac7d00

[17] L. Weinberg, N. Scurrah, K. Gunning and L. McNicol, "Postoperative Changes in Prothrombin Time Following Hepatic Resection: Implications for Perioperative Analgesia," Anaesthesia and Intensive Care, Vol. 34, No. 4, 2006, pp. 438-443.

[18] F. Shafiq, M. Hamid and K. Samad, "Complications and Interventions Associated with Epidural Analgesia for Postoperative Pain Relief in a Tertiary Care Hospital,"
Middle East Journal of Anesthesiology, Vol. 20, No. 6, 2010, pp. 827-832.

[19] W. Schwenk and B. Schinkel "Perioperative Schmerztherapie,” Der Chirurg, Vol. 82, No. 6, 2011, pp. 539-556. doi:10.1007/s00104-010-2051-y

[20] W. Baulig, K. Maurer, O. M. Theusinger, V. Hinselmann, B. Baulig, D. R. Spahn, et al., "Continuous Elastomeric Pump-Based Ropivacaine Wound Instillation after Open Abdominal Aortic Surgery: How Reliable Is the Technique?" Heart Surgery Forum, Vol. 14, No. 1, 2011, pp. 51-58. doi:10.1532/HSF98.20101089

[21] A. Karthikesalingam, S. R. Walsh, S. R. Markar, U. Sadat, T. Y. Tang and C. M. Malata, "Continuous Wound Infusion of Local Anaesthetic Agents Following Colorectal Surgery: Systematic Review and Meta-Analysis,” World Journal of Gastroenterology, Vol. 14, No. 34, 2008, pp. 5301-5305. doi:10.3748/wjg.14.5301

[22] T. R. Yoost, M. McIntyre and S. J. Savage, "Continuous Infusion of Local Anesthetic Decreases Narcotic Use and Length of Hospitalization after Laparoscopic Renal Surgery,” Journal of Endourology, Vol. 23, No. 4, 2009, pp. 623-626. doi:10.1089/end.2008.0586 